\title{
Self-Assembly of PS- $b$-PNIPAM- $b$-PS Block Copolymer Thin Films via Selective Solvent Annealing
}

\author{
Merve Cetintas and Marleen Kamperman* \\ Physical Chemistry and Soft Matter, Wageningen University \& Research \\ Stippeneng 4, 6708 WE, Wageningen, The Netherlands \\ marleen.kamperman@wur.nl*
}

\begin{abstract}
Block copolymer (BCP) thin films are interesting material systems for nanofabrication since they can form well-defined periodic nanostructures by microphase separation. However, attaining a specific morphology with the required orientation can be challenging. In this study, we investigated the morphological behavior of polystyrene- $b$-poly $(N$-isopropylacrylamide $)-b$ polystyrene (PS- $b$-PNIPAM- $b$-PS) BCP thin films by using Atomic Force Microscopy (AFM) and in-situ Grazing-Incidence X-ray Scattering (GISAXS) during selective solvent annealing. Thin films of a lamellar BCP were annealed by using various solvents with different selectivity for the blocks, such as PNIPAM-selective methanol, non-selective tetrahydrofuran (THF) and PS-selective toluene. Solvent annealing using methanol: THF 1:2 (v:v) or methanol: toluene 1:1 (v:v) resulted in the formation of hexagonally ordered perpendicular cylinders, whereas no sustained long-range order was found when only one type of solvent was used. PS- $b$-PNIPAM- $b$-PS BCP thin films that have hexagonally ordered perpendicular cylinders are promising for applications where thermo-responsiveness is desired, such as nanofiltration and biomedical applications.
\end{abstract}

Keywords: block copolymer self-assembly, selective solvent annealing, hexagonally ordered perpendicular cylinders, PS- $b$-PNIPAM- $b$-PS block copolymer 


\section{Introduction}

Block copolymer (BCP) thin films having a hexagonally ordered cylindrical morphology, with the cylinders oriented perpendicular to the substrate, are attractive for many applications such as nanoporous membranes [1], pattern transfer [2] and nanolithography [3]. These applications often require that the films are well-ordered and maintain perpendicular orientation over large areas, which can be obtained by using annealing techniques. The most widely used annealing techniques are thermal and solvent annealing. In these techniques, either the temperature of the thin film is increased over the glass transition temperature $\left(T_{g}\right)$ of the blocks or solvent vapor acts as a plasticizer on the BCP and reduces the $T_{g}$ of the blocks below room temperature which causes an increase in chain mobility. As a consequence, the lateral ordering of the $\mathrm{BCP}$ microdomains is significantly promoted. Although thermal annealing is an effective method to create ordered morphologies [4-11], application of high temperatures to BCP thin films may result in the degradation of one or more blocks, causing the final morphology to deteriorate [12-20]. This can also bring a loss to the long-range ordering of the morphology $[16,17,21]$.

Solvent annealing, does not pose any risk of polymer degradation, is faster [22, 23] and introduces structures which are not approachable using thermal annealing [18-20, 24-27]. Solvent annealing also offers the possibility to choose solvents selective for one of the blocks in the copolymer $[19,26,28-36]$. A selective solvent swells one block more than the other block(s) causing an increase in the effective volume fraction of that block during the annealing process. In this way, it is possible to shift through the BCP phase diagram to different regions which correspond to different morphologies without changing the molecular weight or block ratio of the copolymer [20, 23, 37, 38]. Thus, selective solvent annealing is a useful approach to achieve non-equilibrium morphologies without the need of more than one BCP [35]. For example, Chavis et al. used one poly(2-hydroxyethyl methacrylate)- $b$ - 
poly(methyl methacrylate) (PHEMA- $b$-PMMA) BCP to create four different morphologies including hexagonally ordered cylinders by using selective solvent annealing [31]. Precise control of selective solvents was also used to shift to different morphologies during annealing of polystyrene- $b$-poly(2-vinylpyridine) (PS-P2VP) BCPs as reported by Park et al. [33]

An additional interesting feature of using selective solvents is that the orientation of the cylindrical morphologies may be modified [26, 36, 38-40]. For PS- $b$-P4VP BCPs, parallel cylinders were obtained using the non-selective solvent chloroform whereas cylinders oriented perpendicularly in case of using the PS block selective solvent 1,4-dioxane [38, 39]. Brendel et al. showed a perpendicular cylindrical morphology for a semiconductor BCP by annealing with a selective solvent which was not possible to attain with thermal annealing methods [40]. Similarly, Berezkin et al. reported that a certain selectivity of the annealing solvents is necessary for perpendicular orientation of the cylinders while thermal annealing was only able to produce parallel cylinders [26].

In this work, we study the effect of selective solvent annealing on a stimuli-responsive BCP system. Stimuli-responsive thin films are used in various applications such as stimuliresponsive nanoporous membranes [41], chemical sensors for microelectromechanical systems (MEMS), drug release systems, actuators in biomedical applications [42, 43], nanolithography [44] and cell adhesion [45]. Stimuli-responsive thin films are often prepared by grafting a stimuli-responsive polymer to the surface. However, the use of BCPs, containing one or more responsive blocks, forms an interesting alternative [43].

There exist several solvent annealing studies of BCPs containing $\mathrm{pH}$ responsive blocks, including polyacrylic acid (PAA) [46], P2VP and P4VP [28, 33, 38, 47-51], and $\mathrm{pH}$ and temperature dual-responsive blocks, including $\operatorname{poly}(N, N$ '-dimethylaminoethylmethacrylate) (PDMAEMA) and poly( $N, N$ '-diethylaminoethylmethacrylate) (PDEAEMA) [52]. One of the 
most widely used thermo-responsive blocks in BCPs is poly( $N$-isopropylacrylamide) (PNIPAM) which has a lower critical solution temperature (LCST) at $32{ }^{\circ} \mathrm{C}$. Thomas et al. investigated the morphological properties of bulk films of protein-PNIPAM BCPs using selective solvent annealing [53]. In addition, PS-PNIPAM BCPs were successfully used for thermoresponsive nanofiltration purposes [1], cell adhesion and growth studies for biomedical applications [54]. However, to the best of our knowledge, there is no systematic study for solvent annealing for PS- $b$-PNIPAM- $b$-PS BCP thin films using selective solvent systems.

In our study, we fabricated PS- $b$-PNIPAM- $b$-PS BCP thin films which were annealed using various selective solvents. The morphologies of the thin films were analyzed using Atomic Force Microscopy (AFM) and in-situ Grazing Incidence X-ray Scattering (GISAXS) techniques. We show that by solvent annealing in methanol-THF or methanol-toluene solvent mixtures, a hexagonally ordered cylindrical morphology, with the cylinders oriented perpendicular to the substrate, can be obtained from a lamellar BCP thin film. Having the ability to control the order and to maintain the perpendicular orientation of temperatureresponsive $\mathrm{BCP}$ thin films is particularly promising for membrane applications.

\section{Experimental Part}

\subsection{Materials}

Styrene (Sigma-Aldrich, >99\%) was vacuum distilled after stirring overnight over calcium hydride. $\quad N$-isopropylacrylamide (NIPAM, Sigma-Aldrich, 97\%) was purified by recrystallization from toluene. 2,2'-Azobis(2-methylpropionitrile) (AIBN, Sigma-Aldrich, 98\%) was recrystallized from methanol. Diethylether (Biosolve), methanol (Fisher Scientific), tetrahydrofuran (THF), 1,4-dioxane and toluene (Sigma-Aldrich) were used without further purification. 


\subsection{Synthesis}

2.2.1. Synthesis of PS macro-RAFT agent: A difunctional RAFT agent S,S'-bis $\left(\alpha, \alpha^{\prime}-\right.$ dimethyl- $\alpha$ "-acetic acid) trithiocarbonate (BDAT) was synthesized by using the method reported by Lai et al. [55] and characterized by ${ }^{1} \mathrm{H}$ NMR and ${ }^{13} \mathrm{C}$ NMR. The synthesis of the PS macro-RAFT agent and the copolymer were based on the procedure reported by Nykänen et al. [1]. BDAT (0.7mM, $14 \mathrm{mg})$, freshly distilled styrene (2.2 M, $16 \mathrm{~g})$ and AIBN (0.8mM, $9.2 \mathrm{mg}$ ) were dissolved in $70 \mathrm{~mL}$ of 1,4-dioxane by stirring at room temperature. The solution was transferred to a Schlenk ampoule by using syringes. After degassing with three successive freeze-pump-thaw cycles by using a high vacuum Schlenk line, the solutions were heated to $70^{\circ} \mathrm{C}$ with a temperature controlled oil bath. The solution was allowed to polymerize for 48 hours. The reaction was stopped by cooling the ampoules in liquid nitrogen. The product was precipitated twice in cold methanol and dried in a vacuum oven at room temperature overnight to give a white-yellowish powder (22\% yield). ${ }^{1} \mathrm{H} \mathrm{NMR}\left(\mathrm{CDCl}_{3}\right)$ : 6.2-7.1 (Ar-5H), 1.2-2.4 (3H, PS backbone). GPC: $M_{n}=57 \mathrm{kDa}, \mathrm{PDI}=1.40$.

2.2.2. Synthesis of PS-b-PNIPAM-b-PS copolymers [1]: The PS macro-RAFT agent (3 g, 1.2mM), NIPAM (6 g, 0.9 M) and AIBN (1.99 mg, $0.206 \mathrm{mM})$ were dissolved in $60 \mathrm{~mL}$ of 1,4-dioxane. The solution was degassed by three successive freeze-pump-thaw cycles and heated in a temperature controlled oil bath to $70^{\circ} \mathrm{C}$ for $18 \mathrm{~h}$. The reaction was stopped by cooling the reaction mixture in liquid nitrogen. 1,4-dioxane was removed with a rotary evaporator after which the $\mathrm{BCP}$ was dissolved in THF. The $\mathrm{BCP}$ was reprecipitated from diethyl ether and cold water, respectively. The precipitant obtained from diethyl ether precipitation was separated by centrifuging with PTFE centrifuge tubes and decantation. The product was separated from the homopolymer PNIPAM by centrifugation (45 min, $5000 \mathrm{rpm}$ ) three times. The purified copolymer was freeze-dried giving $41 \%$ yield. The molar ratio of the PS:PNIPAM blocks is equal to 0.90 which was calculated from the NMR data. This 
corresponds to a PS weight fraction 0.45 and a PS volume fraction $\left(f_{\mathrm{PS}}\right)$ of 0.44 (using $\rho_{P S}=$ $1.05 \mathrm{~g} / \mathrm{cm}^{3}[56]$ and $\left.\rho_{\text {PNIPAM }}=1.10 \mathrm{~g} / \mathrm{cm}^{3}[57]\right) .{ }^{1} \mathrm{H}$ NMR $\left(\mathrm{CDCl}_{3}\right): 6.2-7.1(\mathrm{Ar}-5 \mathrm{H}), 4.0(1 \mathrm{H}$, NCH-), 0.8-2.5 (3H, PS backbone and 9H, PNIPAM $-\mathrm{CH}_{3}$ and the backbone). GPC: $M_{n}=$ $127 \mathrm{kDa}, \mathrm{PDI}=1.40$.

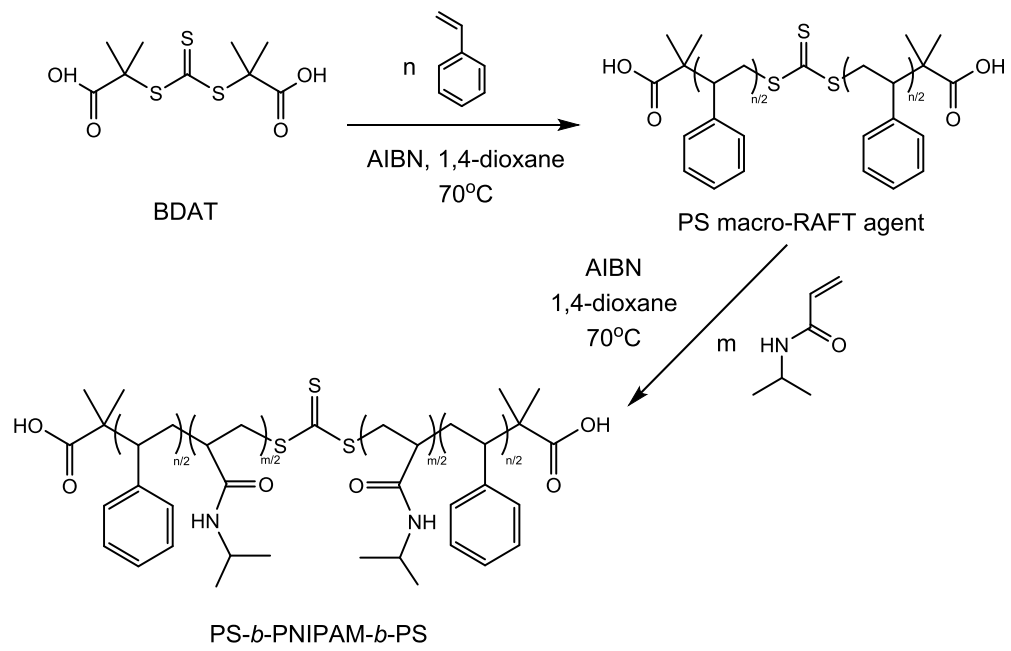

Scheme 1. Synthesis route of PS- $b$-PNIPAM- $b$-PS BCP

\subsection{Polymer Characterization}

${ }^{1} \mathrm{H}$ NMR measurements were carried out on a Bruker AMX-400 spectrometer $(400 \mathrm{MHz})$ at room temperature. The ratio between PS and PNIPAM blocks in the BCP was determined by comparing the integral of the aromatic PS protons at 6.2-7.1 $\mathrm{ppm}(5 \mathrm{H}, \mathrm{Ar}-\mathrm{H})$ to the lone PNIPAM proton at $4.0 \mathrm{ppm}(1 \mathrm{H},-\mathrm{NCH})$. Gel permeation chromatography (GPC) of the polymers was carried out by using a set-up consisting of an Agilent Technologies 1200 series gel permeation chromatograph (GPC), a PLgel $5 \mu \mathrm{m}$ Mixed-D column (Mw range 200-400.000 Da, Polymer Laboratories Ltd.) and an Agilent 1200 differential refractometer. The column was calibrated by using PS standards. An amount of $100 \mu \mathrm{L}$ of each sample was injected into the $\mathrm{THF}$ eluent at $30{ }^{\circ} \mathrm{C}$ and a flow rate of $1 \mathrm{~mL} / \mathrm{min}$. 


\subsection{Bulk Film Preparation}

A 3wt $\%$ copolymer solution in THF was prepared and stirred overnight for full dissolution. The solution was poured into a PTFE beaker. This beaker was transferred to a desiccator, containing a beaker full of THF, which forms the desired solvent atmosphere, and solvent annealed for 2 weeks. After annealing, the THF beaker was taken out and transferred to a vacuum oven where vacuum was applied slowly for 6 hours at room temperature. Then the temperature of the vacuum oven was increased stepwise first to $40^{\circ} \mathrm{C}$ and then to $60^{\circ} \mathrm{C}$. The beaker was kept under vacuum at $60^{\circ} \mathrm{C}$ for at least 18 hours. Then cooled to room temperature after which the vacuum was released, yielding a solid film without any air bubbles.

\subsection{Thin Film Preparation}

Films were deposited from $2 \mathrm{wt} \%$ solutions of copolymer by spin-coating on $\mathrm{Si}$ wafers at $3500 \mathrm{rpm}$ for 15 seconds with a Laurell WS-650MZ-23NPP spin-coater in $\mathrm{N}_{2}$ atmosphere. $1 \times 1 \mathrm{~cm}$ and $2 \times 2 \mathrm{~cm}$ sized $\mathrm{Si}$ wafers were used for solvent annealing experiments in the desiccator and for in-situ GISAXS experiments, respectively. Solvent annealing was stopped by fast quenching, which was executed by opening the cap of the desiccator and removing all the solvent from the desiccator. Si wafers were pre-treated with piranha solution (attention: highly oxidizing!) and stored in a methanol-water solution and rinsed with acetone just before use. THF was used as the solvent for the preparation of the solutions for spin-coating unless stated otherwise. The thickness of the films was measured with spectroscopic ellipsometry (Sentech Instruments $\mathrm{GmbH}$ ) at an incidence angle of $70^{\circ}$ with a wavelength of $632.8 \mathrm{~nm}$ and was found to be around $100 \mathrm{~nm}$. The spin-coated films that were not used for in-situ GISAXS experiments were annealed at room temperature in a desiccator saturated with solvent or with a solvent mixture for a certain amount of time. 


\subsection{Atomic Force Microscope (AFM)}

The surface morphology of the films was analyzed with a Bruker Multimode 8 AFM instrument using the Nanoscope V ScanAsyst imaging mode. DNP-10 model non-conductive silicon nitride probes with a spring constant of $0.24 \mathrm{~N} / \mathrm{m}$ (Bruker) were used. Images were recorded at a frequency of $1.50 \mathrm{~Hz}$ and NanoScope Analysis 1.5 software was used for the processing of the data. At least three different regions on the same thin film sample were probed to assure that the obtained surface morphology was representative for the entire sample.

\subsection{Small Angle X-ray Scattering (SAXS)}

Small angle X-ray scattering measurements were performed on a SAXSLAB GANESHA 300 XL SAXS system equipped with a GeniX 3D Cu Ultra Low Divergence micro focus sealed tube source. The wavelength $\lambda$ was $1.54 \AA$ at a flux of $1 \times 10^{8}$ photons/s. A Pilatus $300 \mathrm{~K}$ silicon pixel detector with $487 \times 619$ pixels and a size of $172 \times 172 \mu \mathrm{m}$ was placed at a sample-to-detector distance of $1513 \mathrm{~mm}$. Silver behenate was used for calibration of the beam centre and the $q$-range. The calibrated detector response function was used together with the known sample-to-detector distance, measured incident and transmitted beam intensities, to bring the two-dimensional SAXS patterns to an absolute intensity scale. The corrected SAXS patterns were azimuthally averaged to obtain one dimensional SAXS profiles. SAXSGUI v2.13 software was used to analyze the data.

\subsection{Grazing Incidence Small Angle X-ray Scattering (GISAXS)}

In-situ GISAXS experiments were carried out at beamline D1 at the Cornell High Energy Synchrotron Source (CHESS) at Cornell University, Ithaca, New York, USA. The wavelength 
$\lambda$ was $0.1162 \mathrm{~nm}$ and the beam size $0.5 \mathrm{~mm} \times 0.1 \mathrm{~mm}$. A CCD camera with a pixel size of $46.9 \mu \mathrm{m}$ was used as detector and placed at a sample-to-detector distance of $1825 \mathrm{~mm}$. The sample was placed in a custom-made annealing chamber having a volume of $110 \mathrm{~mL} .3 \mathrm{~mL}$ of solvent was injected into the chamber. Two or three exposures were taken before injection of the solvent and every 5 minutes during the annealing process. The sample was being moved to a previously unexposed area after around 10 exposures to avoid beam damage of the sample. The annealing chamber was connected to a flowmeter which was used to control the evaporation rate of the solvent inside the chamber [58]. It was equipped with a FilMetrics F30 optical spectroscopic reflectometer for monitoring the thickness of the films during the annealing process. The spots where the thickness was measured were not exposed to X-rays.

ProcessGIXS 8 software was used to analyze the data. Error bars in Figure 7 and Figure 10 were calculated by taking the standard deviation between the second order polynomial fit and the raw data of around 20 data points. Since the resulting error bars were smaller than the size of the marker on the graph, they were not plotted in the Figures.

\section{Results and Discussion}

In this work, we aim to fabricate PS- $b$-PNIPAM- $b$-PS thin films having a cylindrical morphology with hexagonal perpendicular alignment by using solvent annealing. We explore the effect of several selective solvents and combinations of them on the ordering of the BCP thin films. Common laboratory solvents were selected, i.e. THF, methanol and toluene. The vapor pressures of all solvents are similar except for toluene, which has a lower vapor pressure as shown in Table 1 [59]. Since slow drying of the swollen film will often lead to changes in the final morphology of the block copolymer thin film [27], we rapidly quenched the films to kinetically trap the morphology [58]. 
To compare the selectivity of these solvents for the blocks of the copolymer, we calculated the Flory interaction parameters $(\chi)$ using Hansen solubility parameters. $\chi$ parameters were calculated using equations 1 and 2 [60].

$$
A_{1,2}=\left[\left(\delta_{D 2}-\delta_{D 1}\right)^{2}+0.25\left(\delta_{P 2}-\delta_{P 1}\right)^{2}+0.25\left(\delta_{H 2}-\delta_{H 1}\right)^{2}\right]
$$

In equation $1, \delta_{\mathrm{D}}, \delta_{\mathrm{P}}$ and $\delta_{\mathrm{H}}$ are Hansen solubility parameters for dispersive, polar and hydrogen bonding contributions of the two blocks of the BCP, respectively [60, 61]. Subscript 1 stands for the solvent and 2 stands for the polymer block.

$$
\chi_{12}=V A_{1,2} / R T
$$

In equation $2, V, R$ and $T$ correspond to the molar volume of the solvent, the ideal gas constant and the absolute temperature, respectively. The calculated results, given in Table 1, indicate that methanol is a better solvent for PNIPAM than for PS, while toluene is a better solvent for PS. THF can be considered as a neutral solvent for both blocks. Although some of the calculated $\chi$ values were significantly higher than experimental values reported in the literature, for our experiments it was sufficient to compare the selectivity of the solvents between the blocks.

Table 1. Calculated $\chi$ parameters and vapor pressures

\begin{tabular}{|l|l|l|l|l|}
\hline \multicolumn{2}{|l|}{} & methanol & THF & toluene \\
\hline$\chi$ parameters & PS & 2.14 & 0.78 & 0.74 \\
& PNIPAM & 1.13 & 0.46 & 1.21 \\
\hline \multicolumn{2}{|l|}{ Vapor pressure at $25^{\circ} \mathrm{C}(\mathrm{kPa})$} & 16.9 & 21.6 & 3.79 \\
\hline
\end{tabular}




\subsection{Bulk Morphology}

The triblock PS- $b$-PNIPAM- $b$-PS copolymer with a total molecular weight of $M_{n}=127 \mathrm{kDa}$, $\mathrm{PDI}=1.40$ and $f_{\mathrm{PS}}=0.44$ was successfully synthesized by RAFT polymerization (M. Cetintas et al. unpublished results). The bulk morphology of the copolymer was characterized by using SAXS and AFM, after solvent annealing of the bulk copolymer film. As shown in Figure 1a, the intensity profile of the SAXS data shows a well-defined first-order peak and a higher order reflection at a $q$ spacing ratio of $3 q^{*}$. The AFM image, shown in Figure $1 \mathrm{~b}$, shows a lamellar morphology perpendicularly aligned to the surface with an average interlayer distance of $59+/-2 \mathrm{~nm}$. This interlayer distance is in agreement with the SAXS data from which an interlayer distance of $59.3 \mathrm{~nm}$ was obtained. In the SAXS traces, no clear second order peak at $q$ spacing ratio of $2 q^{*}$ was observed, which can indicate that the lamellae of the two blocks are similar in thickness [62]. This is consistent with the almost equal volume fractions of the polymer blocks. 

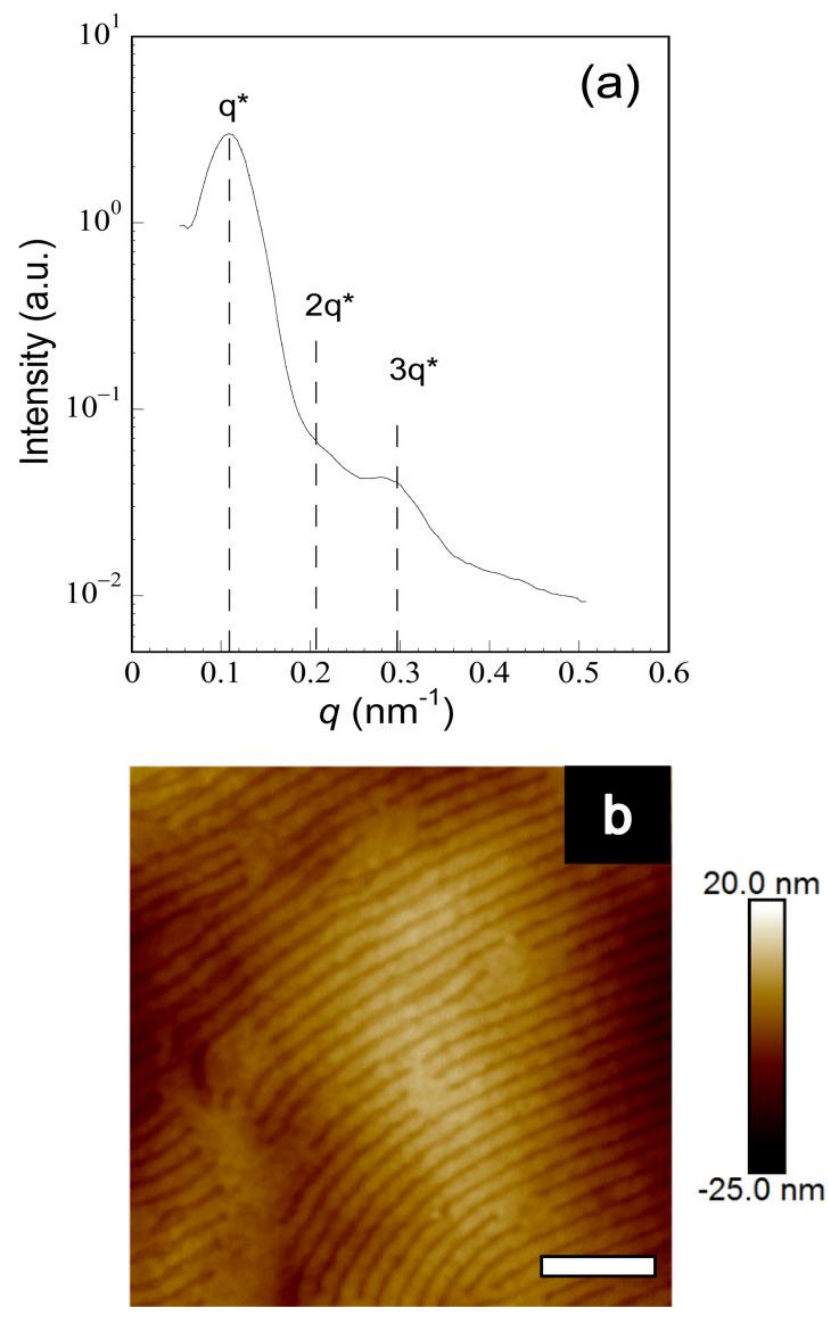

Figure 1. Bulk film characterization. (a) Intensity profile of the SAXS data. Peak positions as expected for a lamellar morphology are indicated. (b) AFM height image. Scale bar $=400 \mathrm{~nm}$.

\subsection{As-spun samples}

Copolymer thin films with a thickness of around $100 \mathrm{~nm}$ were prepared by spin-coating from PS- $b$-PNIPAM- $b$-PS solutions in THF or methanol: THF $(2: 1 \mathrm{v}: \mathrm{v})$ solvent mixtures. The variation in thickness for a set of samples annealed with a particular solvent (mixture) was $+/-$ $10 \mathrm{~nm}$. Methanol is a more selective solvent for PNIPAM than for PS. THF is almost neutral for both blocks. The AFM images in Figure 2 show a disordered micellar morphology for both types of solvent systems. The light regions in the images correspond to PS and the dark regions correspond to the PNIPAM part of the block copolymer. The micelles can either 
indicate spheres or perpendicular cylinders. The GISAXS images in Figure SI show a weak first-order peak and confirm that there is only short-range ordering in the lateral direction with domain spacings of $62.8 \mathrm{~nm}$ for $\mathrm{THF}$ and $40 \mathrm{~nm}$ for methanol/THF. This relatively large difference in domain size, may be due to the poor solubility of PS in methanol. Since the obtained as-spun morphologies are metastable due to the rapid solvent evaporation during spin-coating, collapse of the PS chains in solution may cause a decrease in domain size. Both the AFM and the GISAXS data indicate that the methanol-THF solvent mixture slightly improved the uniformity of the domains. Therefore, the use of selective solvents improved the morphology [51].
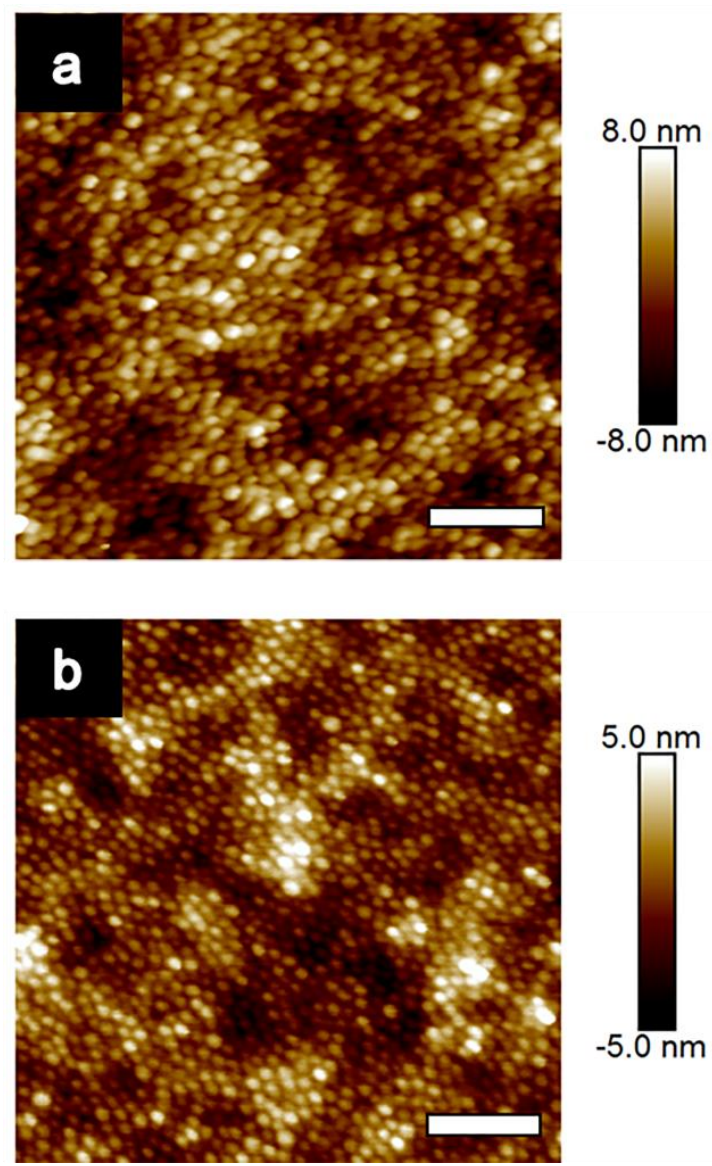

Figure 2. AFM topography images of as spun thin films prepared by spin coating solutions of the BCP in (a) THF and (b) methanol-THF. Scale bar $=400 \mathrm{~nm}$. 


\subsection{Solvent Annealing}

\subsubsection{Solvent annealing with pure solvents}

We solvent annealed as-spun BCP thin films using three different pure solvents: THF, methanol and toluene, and studied the morphological behavior using in-situ GISAXS and AFM. The GISAXS analysis was performed on films swollen with solvent and AFM analysis on quenched dry thin films after solvent annealing. The as-spun morphology for solvent annealing was always disordered micellar as shown in Figure 2. In all solvent annealing experiments, including with pure solvents and solvent mixtures, there was always an initial first order peak in the dry state which immediately disappeared when it came into contact with the solvent vapor. The occurrence and disappearance of this initial peak will not be mentioned for each system separately.

Toluene is a good solvent for PS, but a poor solvent for PNIPAM. For toluene, a weak first order peak was observed for a short time, after which the film became and stayed disordered during the solvent annealing process (Figure 3a). GISAXS images of the integrated plots in Figure 3 can be found in Figure SII. While the thickness of the film increased by $50 \%$ (from 100 to $150 \mathrm{~nm}$ ) there was apparently insufficient plasticization to induce any reordering of the BCP domains. The initial weak first order peak may be lost due to a small screening effect by the toluene of the non-favorable interactions between the blocks. 

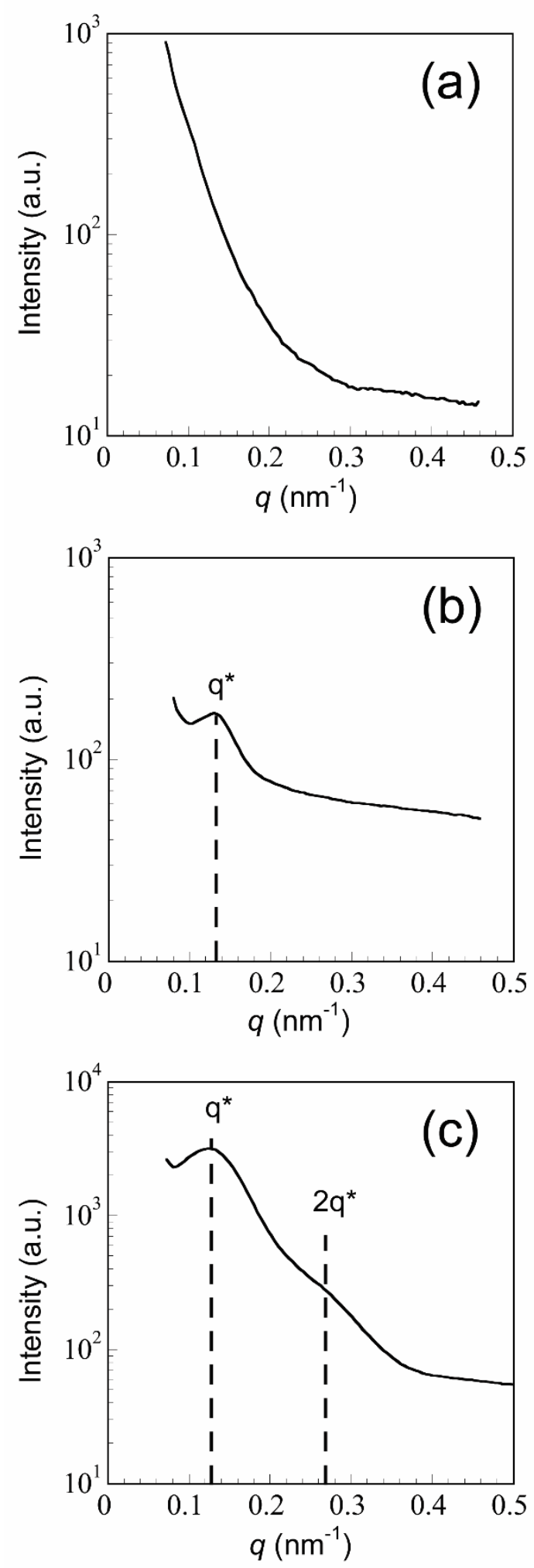

Figure 3. In-plane GISAXS profiles obtained during solvent annealing in (a) toluene, (b) THF and (c) methanol vapor. Polymer fractions for toluene, THF and methanol were 0.84, 0.53 and 0.37, respectively. Corresponding 2D GISAXS data can be found in Figure SII. 
For both THF and methanol annealing, a stable and well-defined first order peak was obtained, as can be seen in Figure $3 \mathrm{~b}$ and 3c, respectively. For THF the film could be swollen up to $363 \%$ relative to its original film thickness, but no higher order peaks were obtained during the process. Since THF is an almost non-selective solvent for the PS-PNIPAM system increasing the solvent content in the film not only increases the chain mobility but also leads to an increased screening of the non-favorable interactions between the blocks. Therefore, the lack of higher order may also indicate that the solvent concentration during in-situ GISAXS was too high to obtain well-defined morphologies. To complement the swelling data we performed separate AFM studies on solvent annealed films that were rapidly quenched by removing the samples from the annealing desiccator. According to the AFM images in Figure 4, after 60 minutes of THF annealing there was almost no change in the morphology of asspun samples and after 120 minutes, worm-like micelles were formed. After 240 minutes, there was a mixed morphology of parallel lamellae and either parallel cylinders or perpendicular lamellae. Although phase transitions could be observed with AFM, none of the images showed order over large areas, in agreement with the GISAXS data.

For solvent annealing with methanol a final film thickness of $171 \%$ the original film thickness was reached. This value lies between the final thickness of toluene and THF, which is due to a combined effect of vapor pressure and solvent quality of the respective solvents. Methanol has higher interactions parameters with the polymer blocks than THF and toluene, but toluene has a low vapor pressure. At similar conditions (temperature and air flow) the low vapor pressure of toluene results in a lower driving force for the toluene to diffuse into the film as compared to more volatile solvents. In-situ GISAXS data showed that next to a well-defined first order peak, a second order shoulder at $2 q^{*}$ was observed during solvent annealing upon reaching a polymer fraction of 0.47 . The peak intensities increased until a polymer fraction of 0.37 was reached at the end of the experiment. The improved order obtained in GISAXS for 
methanol-annealing as compared to THF-annealing may be explained by the fact that methanol is a more selective solvent than THF and the screening effect of non-favorable interactions will be less pronounced. The complementary AFM study, as shown in Figure 5, only indicated micelles with short-range order even after 2 hours of solvent annealing. Apparently, quenching of the methanol swollen films leads to loss of long-range order.
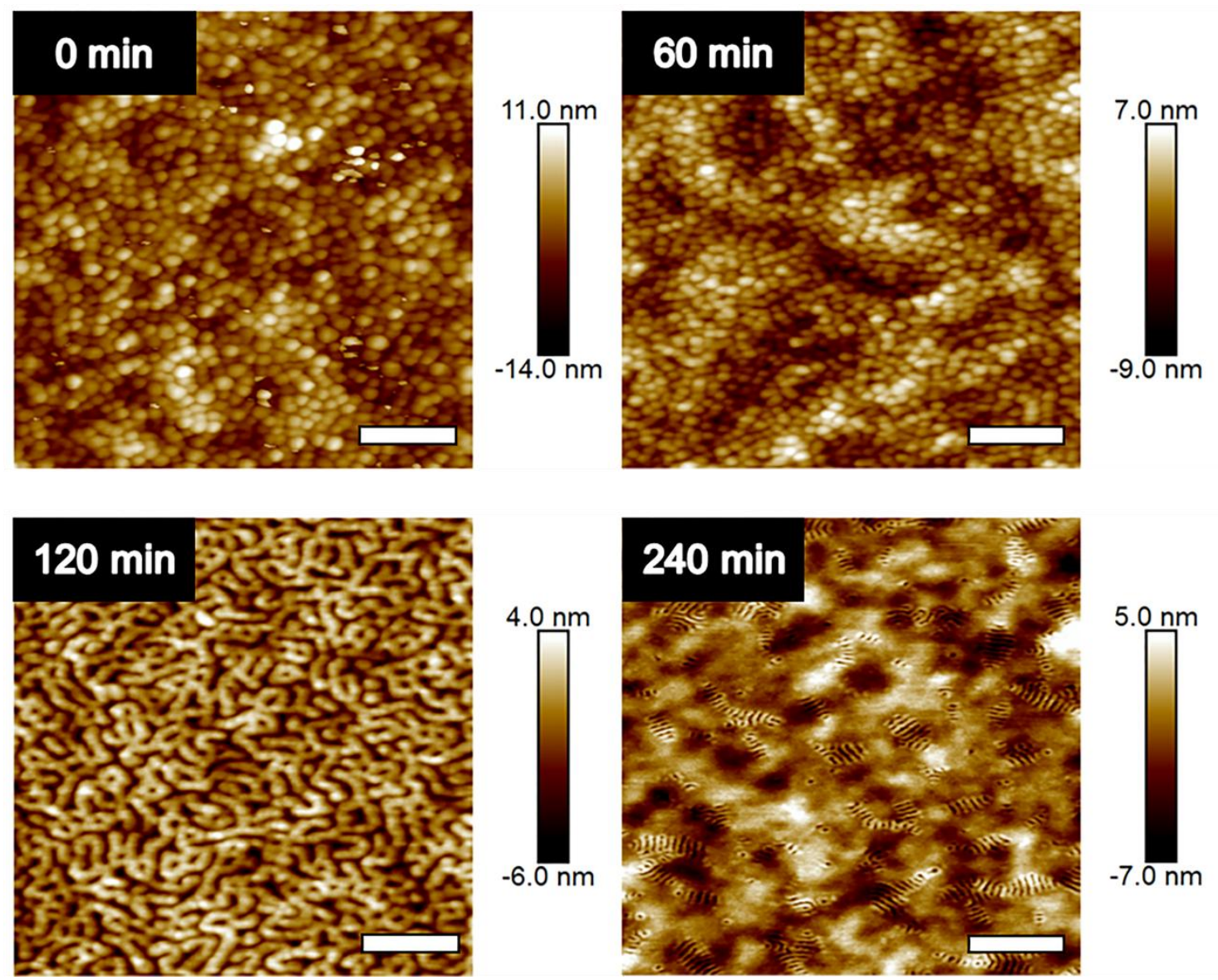

Figure 4. AFM images of time dependent solvent annealing using THF. Scale bar $=400 \mathrm{~nm}$ for 0,60 and 120 minutes and scale bar $=1 \mu \mathrm{m}$ for 240 minutes. 

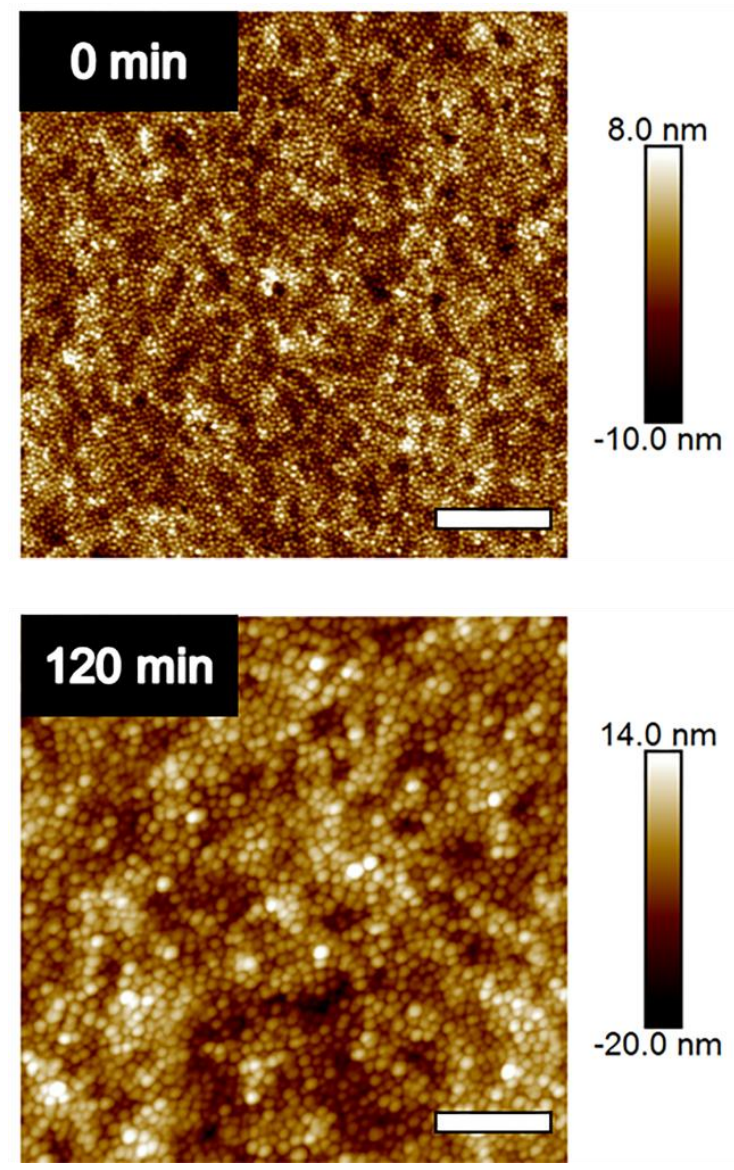

Figure 5. AFM images of time dependent solvent annealing with methanol. Scale bar $=1 \mu \mathrm{m}$ for 0 minutes and scale bar $=400 \mathrm{~nm}$ for 120 minutes.

\subsubsection{Solvent annealing with selective solvent mixtures}

After establishing that no sustained long-range order was found by using pure solvents, we continued with selective solvent mixtures.

\subsubsection{Methanol-THF:}

Figure 6a shows a series of in-plane GISAXS profiles that were collected during annealing of a BCP thin film using a 1:2 (v:v) methanol-THF mixture. The corresponding 2D GISAXS image for a polymer fraction of 0.44 is given in Figure $6 \mathrm{~b}$ and the rest of the $2 \mathrm{D}$ images are given in Figure SIII. During the annealing process the thickness of the film was monitored and Figure 7 shows the domain spacing as a function of the polymer volume fraction $\phi_{\mathrm{BCP}}$. 
The polymer volume fraction is determined by the ratio of the dry polymer film thickness, $t_{0}$, over the swollen film thickness, $t$ :

$$
\phi_{\mathrm{BCP}}=\frac{t_{0}}{t}
$$

Similar as with the pure solvent systems, the as-spun morphology for solvent annealing was always disordered micellar. Upon swelling of the film the first order peak was initially lost. A well-defined first order peak, corresponding to a domain spacing of $55.1 \mathrm{~nm}$, reappeared when the films started to show significant reorganization at a polymer fraction of 0.66 . Higher order reflections at $q$ spacing ratios of $\sqrt{ } 3 q^{*}$ and $\sqrt{ } 7 q^{*}$ started to become visible after reaching a polymer fraction of 0.48 and after the domain spacing had shifted to $57.1 \mathrm{~nm}$. This profile is consistent with hexagonally ordered perpendicular cylinders. The peaks sharpened and intensified upon further swelling (from a polymer fraction of 0.46 to 0.44 ) and the perpendicular orientation of the cylinders was maintained. Due to the selectivity of methanol for PNIPAM it is expected that the PNIPAM forms the majority matrix phase surrounding PS perpendicular cylinders. During the annealing process the thickness of the film increased by $123 \%$ (reaching a polymer volume fraction of 0.44 ) and the domain spacing started at 55.1 and shifted to $69.8 \mathrm{~nm}$. 


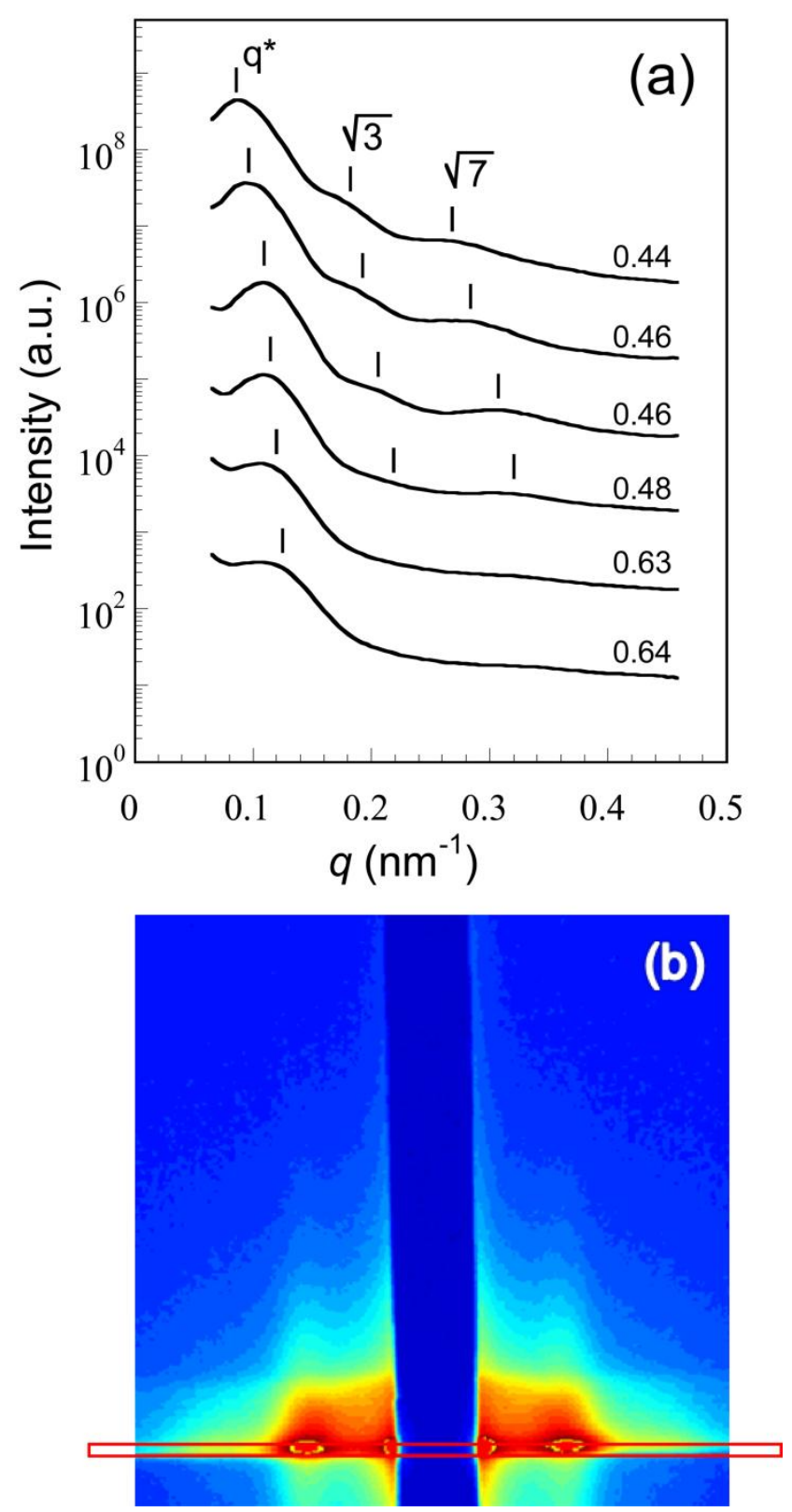

Figure 6. (a) In-plane GISAXS profiles collected during annealing of a BCP thin film using a 1: 2 (v: v) methanol-THF mixture. Polymer volume fractions are indicated next to the profiles. (b) Corresponding 2D GISAXS images for a polymer fraction of 0.44 . The red box indicates the integrated area.

The domain spacing did not constantly increase with polymer fraction, as is indicated in Figure 7. An increasing domain spacing is indicated by green data points and a decrease by red data points. An increase in domain spacing indicates that the film is simply taking up 
more solvent, whereas a decrease in domain spacing (despite the solvent-uptake) usually results from relaxation of the $\mathrm{BCP}$ chain at the interface due to the increase in screening of the non-favorable interactions.

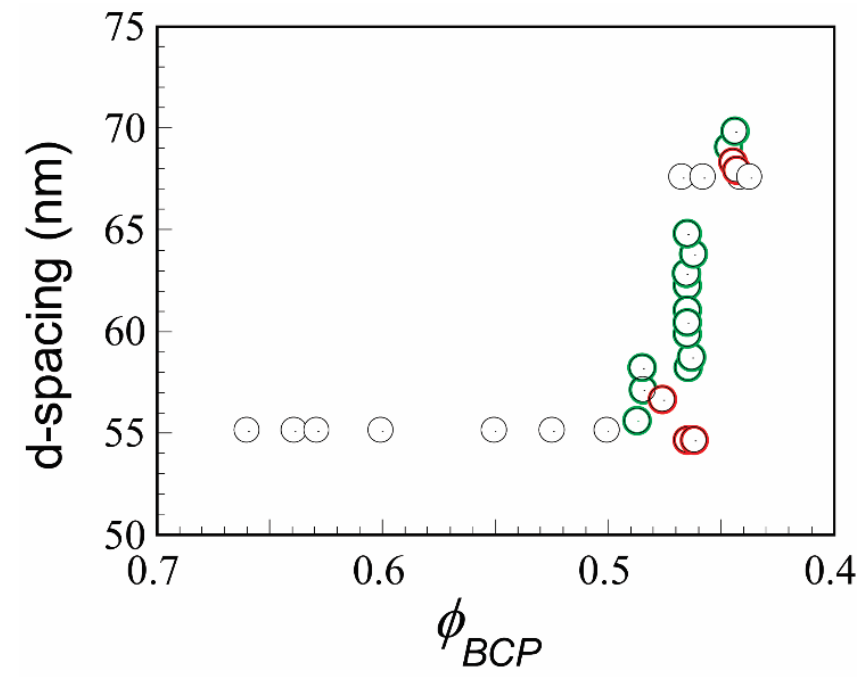

Figure 7. Domain spacing as a function of the polymer volume fraction for methanol:THF 1:2 (v:v) solvent annealing Green data points indicate an increasing trend in d-spacing and red data points a decreasing d-spacing. Error bars are smaller than the size of the markers and are therefore not shown in the graph.

The GISAXS study was again combined with a separate AFM study. Figure 8 shows that disordered micelles started to gain some mobility after 20 minutes of solvent annealing. Ordering improved after 40 minutes and after 70 minutes of solvent annealing, well-defined hexagonally ordered perpendicular cylinders were observed. Upon further increase of the solvent annealing time, it was observed that cylinders tend to align parallel to the substrate. After 120 minutes of solvent annealing, all cylinders showed a parallel alignment. These observations that hexagonally ordered perpendicular cylinders are obtained for short annealing times and cylinders start to align parallel after longer annealing times are in agreement with several reports in the literature [2, 63] However, no change in orientation of the cylinders was observed during the in-situ GISAXS experiments. 

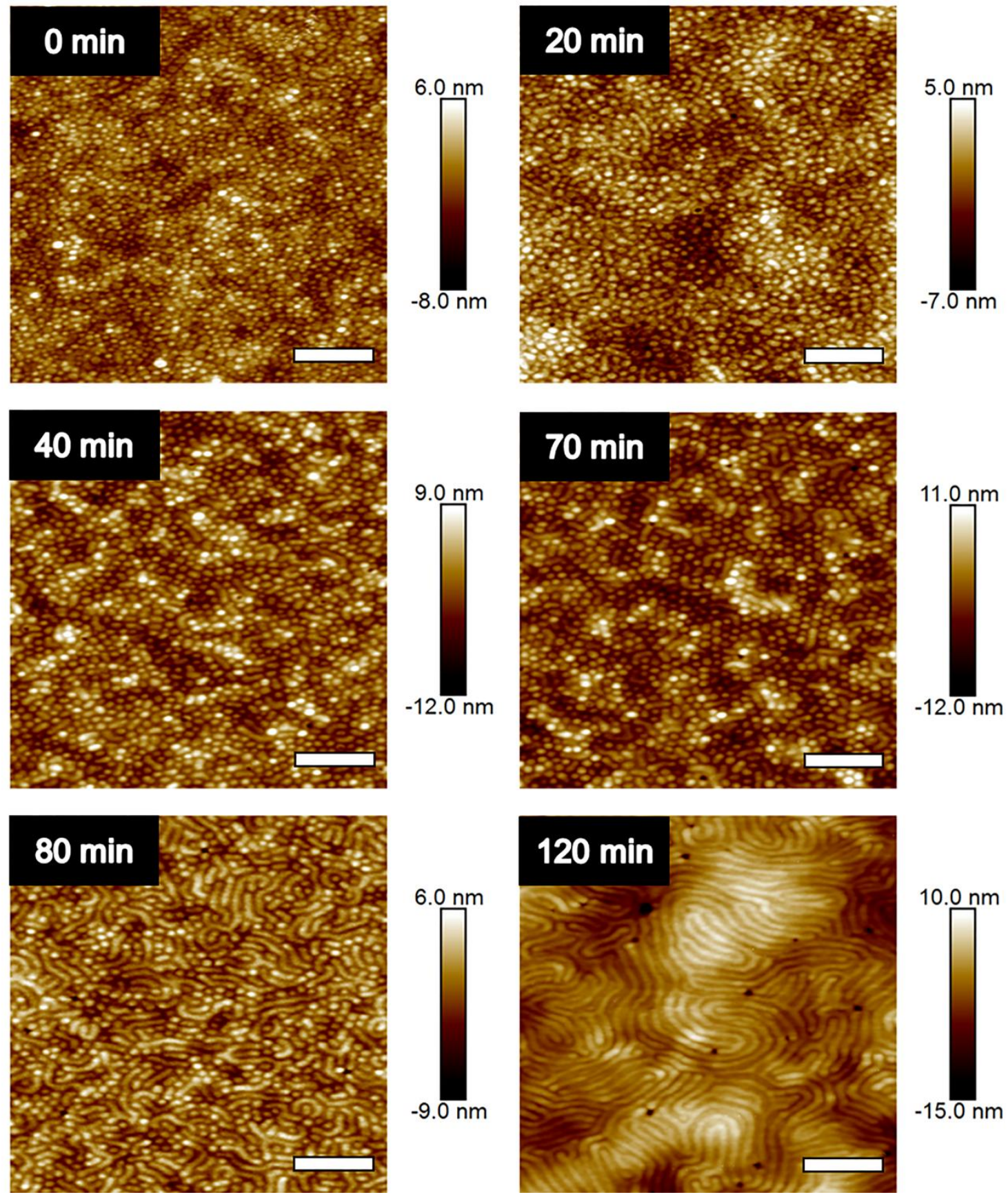

Figure 8. AFM images of time dependent solvent annealing using methanol: THF 1:2 (v:v) solvent mixture. Scale bar $=600 \mathrm{~nm}$.

In addition to varying the annealing time, we also changed the methanol-THF volume ratio and studied the morphological behaviour with AFM after annealing in the vapour of 1:1 and 2:1 (v:v) solvent mixtures (see Figure SIV). Upon increasing the amount of methanol in the solvent mixture from 1: 2 (Figure 8) to 1: 1 (Figure SIV) the morphological rearrangements 
occurred after a longer annealing time. Whereas for the 1: 1 volume ratio, small grains with hexagonally ordered perpendicular cylinders were obtained, for the 2: 1 volume ratio no longrange ordering was observed. The delayed rearrangement of the $\mathrm{BCP}$ chains is consistent with the data of Park et al. where the presence of a poor solvent was also found to increase the annealing time before well-ordered nanostructures were obtained [29]. The reduction in ordering is attributed to the poor solubility of PS in methanol, resulting in a lower mobility of the chains.

\subsubsection{Methanol-toluene:}

Solvent annealing using methanol-toluene mixtures is expected to be considerably different from the use of methanol-THF mixtures, since both solvents are selective. As shown in Table 1, toluene and methanol are selective for PS and PNIPAM, respectively. The volume ratio of methanol: toluene solvent mixture that we used was $1: 1$.

In-situ GISAXS analysis of the annealing process, presented by in-plane profiles in Figure 9a, showed the reappearance of a broad first-order peak after an initial disappearance at the start of the annealing process. The corresponding 2D GISAXS images for a polymer fraction of 0.50 and 0.22 are given in Figure $9 b$ and $9 c$ and the rest of the 2D images are given in Figure SV. The first-order peak reappeared at a polymer volume fraction of 0.55 . A sharpening of the first-order peak and broad higher order reflections at $q$ spacing ratios of $\sqrt{ } 3 q^{*}$ and $\sqrt{ } 7 q^{*}$ started to become visible when the polymer volume fraction was 0.50 . This profile is consistent with hexagonally ordered perpendicular cylinders. However, close inspection of the 2D GISAXS images revealed that the order and orientation of the cylinders did not reach the same level as for methanol-THF annealing. In addition, we noted differences in the 2D GISAXS images in the $q_{z}$-direction at polymer volume ratios of 0.50 and 0.22 (Figure $9 \mathrm{~b}$ and 9c, respectively). The vertical stripes in the 2D GISAXS image of Figure 9b were found to be 
more curved than those of Figure 9c which is an indication that the orientation of the cylinders is not fully perpendicular to the substrate [34].

A surprising feature was the fact that although the polymer volume fraction decreased to 0.22 during the annealing process, the domain spacing of the film did not change significantly and stayed $60.4 \mathrm{~nm}$ during the entire solvent annealing procedure, as shown in Figure 10.

Moreover, at a polymer volume fraction of 0.49 the higher order reflections disappeared and during the course of solvent annealing these peaks reappeared at their original positions at a polymer volume fraction of 0.24 . The disappearance and reappearance of the peaks in the GISAXS profiles may indicate that the system was passed through the order-disorder transition into the disordered state, after which it returned to the ordered state. However, since the solvent concentrations did not decrease during the course of these events, reappearance of the order can only occur if the solvent mixture volume ratio in the film was altered. This may occur, because vapor pressure and solvent quality differences may result in solvent composition variations within the film. At short annealing times, at a polymer volume fraction of 0.50 (see Figure 10), the film will be rich in methanol and will create a more selective environment for PNIPAM, resulting in PS cylinders surrounded by a PNIPAM matrix. At a polymer volume fraction of 0.49 , the higher order peaks disappeared in GISAXS, which indicates that the system rearranged and long-range order was lost. When the polymer volume fraction decreased to 0.24 the ratio of methanol-toluene vapor changed in favor of toluene. We attribute this to the outlet of the solvent chamber, through which more of the solvent with the higher volatility, i.e. methanol, will leave the system. At this point, the amount of toluene in the film may have dominated the amount of methanol, causing the PS domains to swell and the PNIPAM domains to shrink their volume. In the presence of a solvent fraction of 0.76 , the effective volume fraction of PS domains (including the PS selective solvent) can be as large as 0.85 , for which the inverse cylindrical morphology, consisting of PNIPAM cylinders 
within a PS matrix, is expected to be the most stable morphology. This would mean that the morphology is inverted without a change in domain spacing. 

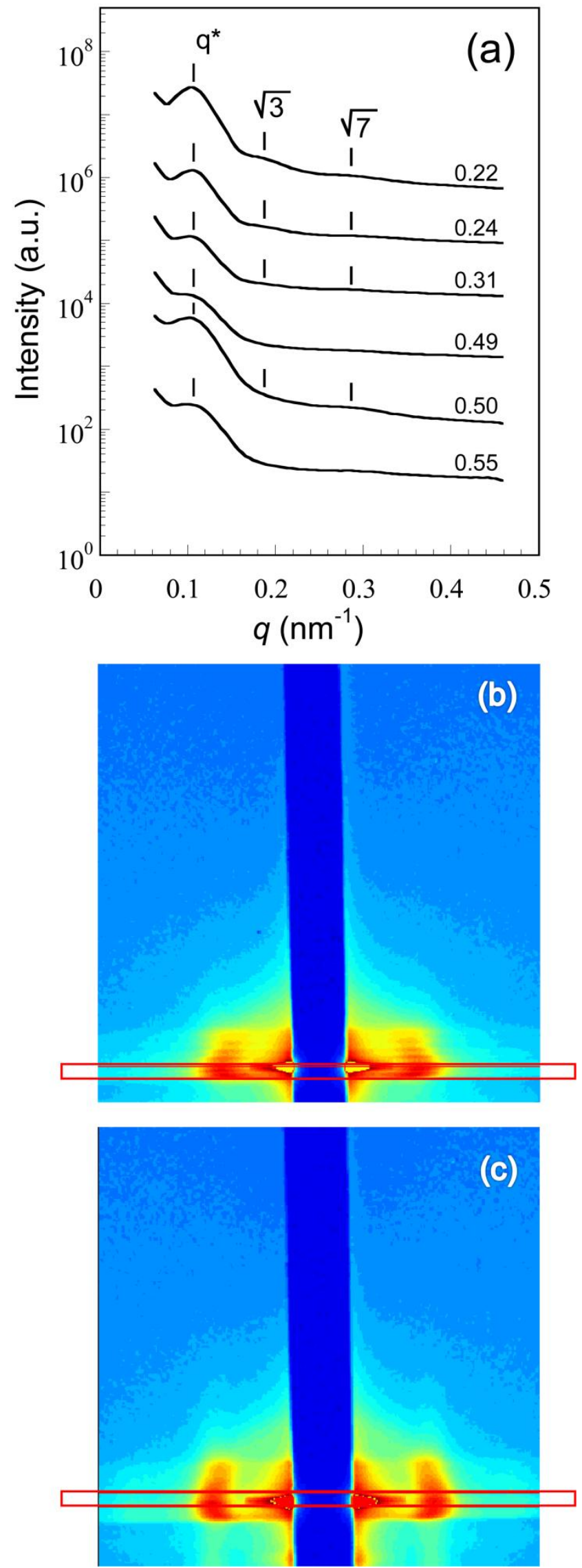
Figure 9. (a) In-plane GISAXS profiles collected during annealing of a BCP thin film using a 1: 1 (v: v) methanol-toluene mixture. Polymer volume fractions are indicated next to the profiles. Corresponding 2D GISAXS images for a polymer volume fraction of (b) 0.50 and (c) 0.22. The red box indicates the integrated area.

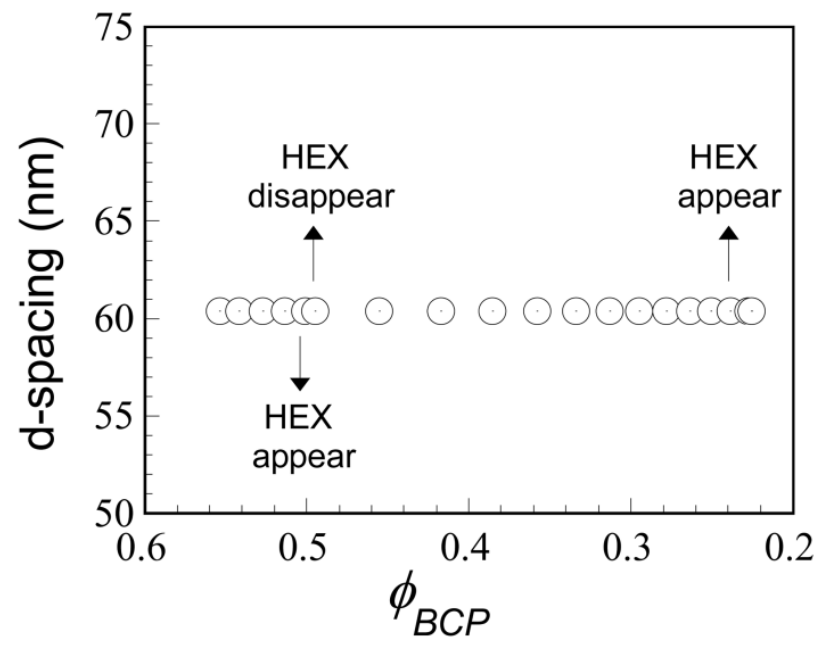

Figure 10. Domain spacing as a function of the polymer volume fraction for methanol:

toluene 1:1 (v:v) solvent annealing. The appearance and the disappearance of the higher order peaks are indicated with arrows. Error bars are smaller than the size of the markers and are therefore not shown in the graph.

The AFM study of quenched morphologies after annealing in the vapor of a 1: 1 (v: v) methanol: toluene mixture is shown in Figure 11. After 40 minutes the micelles started to become mobile. Between 40 and 60 minutes of annealing, a cylindrical morphology was formed with no preferred orientation of the cylinders and only short-range order. After 90 minutes of solvent annealing, however, the cylindrical morphology seemed inverted. As explained above for the GISAXS study this may occur, because vapor pressure and solvent quality differences give rise to solvent composition variations within the films. Even though perpendicular cylinder formation was observed both in GISAXS and in AFM for the 
methanol-toluene solvent annealing system, the ordering did not reach the same level as for methanol-THF solvent annealing.
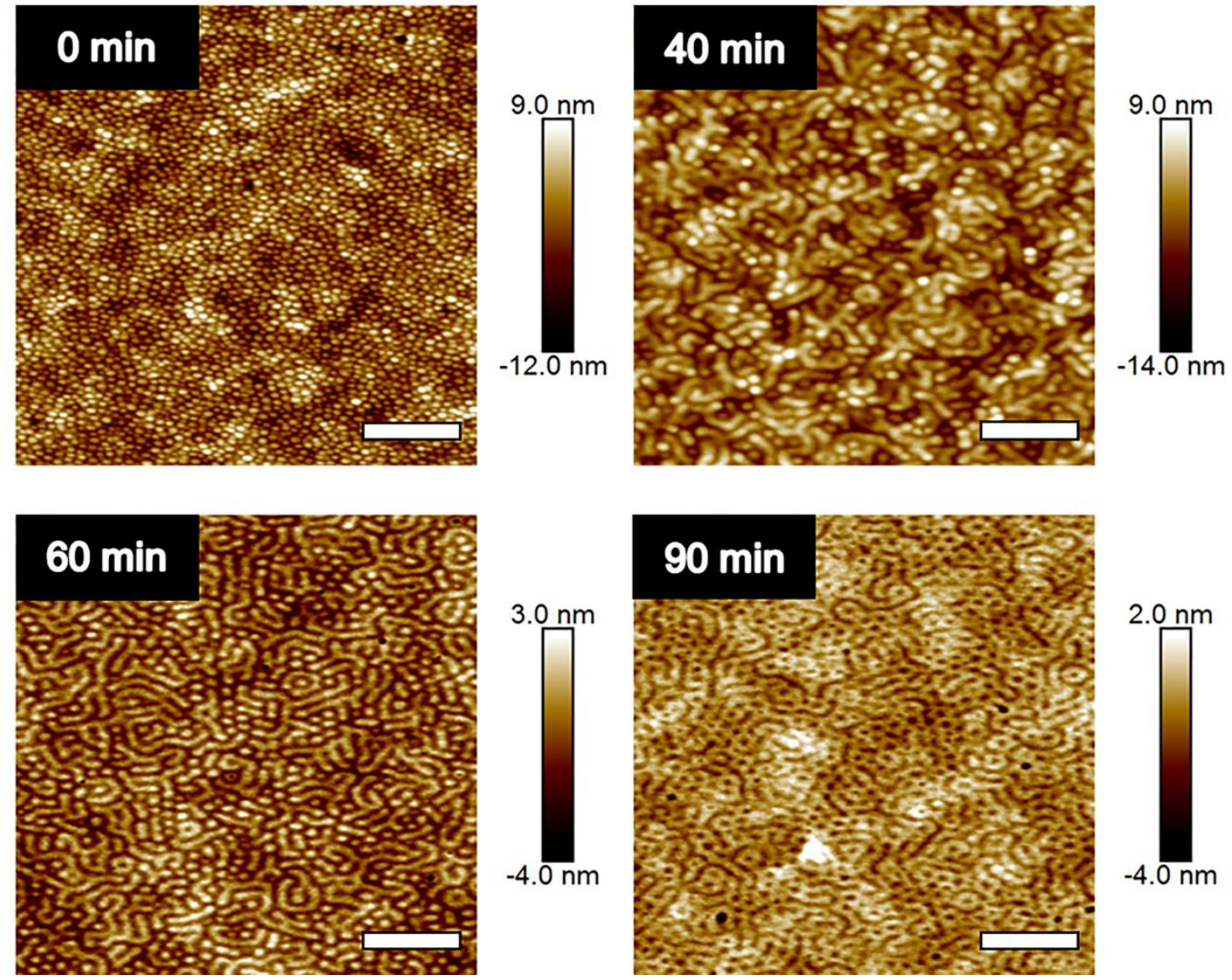

Figure 11. AFM images of time dependent solvent annealing using methanol: toluene 1:1 (v:

v) solvent mixture. Scale bar $=600 \mathrm{~nm}$.

\section{Conclusions}

We studied the morphological behavior of PS- $b$-PNIPAM- $b$-PS BCP thin films upon solvent annealing using selective solvents by means of in-situ GISAXS and AFM. The influence of solvent type, solvent ratio and annealing time were investigated. PS- $b$-PNIPAM- $b$-PS was found to form hexagonally ordered perpendicular cylinders, when annealed with selective solvent mixtures of methanol-THF and methanol-toluene. However, solvent annealing with methanol-THF resulted in a higher degree of order than with methanol-toluene. This 
morphology could not be obtained when only one type of solvent was used, showing the importance of using selective solvents for direct tuning to a specific morphology. PS- $b$ PNIPAM- $b$-PS BCPs hexagonally ordered perpendicular cylinders may find use in BCP thin film applications where thermo-responsiveness is required, such as MEMS, biomedical and nanoporous membrane applications.

\section{Acknowledgements}

The authors are grateful to Detlef-M Smilgies for his help with the GISAXS experiments and Ilja Voets for performing the SAXS experiments. This research forms part of the research programme of the Dutch Polymer Institute (DPI), project \#766 (Dutch Polymer Institute (DPI), P.O. Box 902, 5600 AX Eindhoven, the Netherlands). This work is based upon research conducted at the Cornell High Energy Synchrotron Source (CHESS) which is supported by the National Science Foundation and the National Institutes of Health/National Institute of General Medical Sciences under NSF award DMR-1332208.

\section{Appendix A. Supplementary data}

Supplementary data related to this article is available, free of charge, at http://dx.doi.org/....

\section{References}

[1] A. Nykänen, M. Nuopponen, A. Laukkanen, S.-P. Hirvonen, M. Rytelä, O. Turunen, H. Tenhu, R. Mezzenga, O. Ikkala, J. Ruokolainen, Phase Behavior and Temperature-Responsive Molecular Filters Based on Self-Assembly of Polystyrene-block-poly(Nisopropylacrylamide)-block-polystyrene, Macromolecules 40(16) (2007) 5827-5834.

[2] M. Vayer, M.A. Hillmyer, M. Dirany, G. Thevenin, R. Erre, C. Sinturel, Perpendicular orientation of cylindrical domains upon solvent annealing thin films of polystyrene-bpolylactide, Thin Solid Films 518(14) (2010) 3710-3715.

[3] J. Bang, U. Jeong, D.Y. Ryu, T.P. Russell, C.J. Hawker, Block Copolymer Nanolithography: Translation of Molecular Level Control to Nanoscale Patterns, Adv. Mater. (Weinheim, Ger.) 21(47) (2009) 4769-4792.

[4] H. Jung, S. Woo, Y. Choe, D.Y. Ryu, J. Huh, J. Bang, Single Step Process for SelfAssembled Block Copolymer Patterns via in Situ Annealing during Spin-Casting, ACS Macro Letters 4(6) (2015) 656-660. 
[5] S.Y. Kim, J. Gwyther, I. Manners, P.M. Chaikin, R.A. Register, Metal-Containing Block Copolymer Thin Films Yield Wire Grid Polarizers with High Aspect Ratio, Adv. Mater. 26(5) (2014) 791-795.

[6] C.M. Bates, M.J. Maher, D.W. Janes, C.J. Ellison, C.G. Willson, Block Copolymer Lithography, Macromolecules 47(1) (2014) 2-12.

[7] T.G. Fitzgerald, R.A. Farrell, N. Petkov, C.T. Bolger, M.T. Shaw, J.P.F. Charpin, J.P. Gleeson, J.D. Holmes, M.A. Morris, Study on the Combined Effects of Solvent Evaporation and Polymer Flow upon Block Copolymer Self-Assembly and Alignment on Topographic Patterns, Langmuir 25(23) (2009) 13551-13560.

[8] D.U. Ahn, E. Sancaktar, Fabrication of well-defined block copolymer nano-cylinders by controlling the thermodynamics and kinetics involved in block copolymer self-assembly, Soft Matter 4(7) (2008) 1454-1466.

[9] S.Y. Yang, I. Ryu, H.Y. Kim, J.K. Kim, S.K. Jang, T.P. Russell, Nanoporous Membranes with Ultrahigh Selectivity and Flux for the Filtration of Viruses, Adv. Mater. 18(6) (2006) 709-712.

[10] D.H. Kim, K.H.A. Lau, W. Joo, J. Peng, U. Jeong, C.J. Hawker, J.K. Kim, T.P. Russell, W. Knoll, An Optical Waveguide Study on the Nanopore Formation in Block Copolymer/Homopolymer Thin Films by Selective Solvent Swelling, The Journal of Physical Chemistry B 110(31) (2006) 15381-15388.

[11] U. Jeong, D.Y. Ryu, D.H. Kho, J.K. Kim, J.T. Goldbach, D.H. Kim, T.P. Russell, Enhancement in the Orientation of the Microdomain in Block Copolymer Thin Films upon the Addition of Homopolymer, Adv. Mater. 16(6) (2004) 533-536.

[12] Z. Di, D. Posselt, D.-M. Smilgies, C.M. Papadakis, Structural Rearrangements in a Lamellar Diblock Copolymer Thin Film during Treatment with Saturated Solvent Vapor, Macromolecules 43(1) (2010) 418-427.

[13] J.Y. Kelly, J.N.L. Albert, J.A. Howarter, S. Kang, C.M. Stafford, T.H. Epps, M.J. Fasolka, Investigation of Thermally Responsive Block Copolymer Thin Film Morphologies Using Gradients, ACS Applied Materials \& Interfaces 2(11) (2010) 3241-3248.

[14] R.A. Segalman, Patterning with block copolymer thin films, Materials Science and Engineering: R: Reports 48(6) (2005) 191-226.

[15] V. Abetz, P.F.W. Simon, Phase Behaviour and Morphologies of Block Copolymers, Block Copolymers I, Springer Berlin Heidelberg2005, pp. 125-212.

[16] S.B. Darling, Directing the self-assembly of block copolymers, Progress in Polymer Science 32(10) (2007) 1152-1204.

[17] D.-M. Smilgies, R. Li, Z. Di, C. Darko, C.M. Papadakis, D. Posselt, Probing the SelfOrganization Kinetics in Block Copolymer Thin Films, Materials Research Society Symposium Proceedings 1147E (2009).

[18] J.Y. Kelly, J.N.L. Albert, J.A. Howarter, C.M. Stafford, T.H. Epps, M.J. Fasolka, Manipulating morphology and orientation in thermally responsive block copolymer thin films, Journal of Polymer Science, Part B: Polymer Physics 50(4) (2012) 263-271.

[19] K.W. Gotrik, A.F. Hannon, J.G. Son, B. Keller, A. Alexander-Katz, C.A. Ross, Morphology Control in Block Copolymer Films Using Mixed Solvent Vapors, ACS Nano 6(9) (2012) 8052-8059.

[20] J.K. Bosworth, M.Y. Paik, R. Ruiz, E.L. Schwartz, J.Q. Huang, A.W. Ko, D.-M. Smilgies, C.T. Black, C.K. Ober, Control of Self-Assembly of Lithographically Patternable Block Copolymer Films, ACS Nano 2(7) (2008) 1396-1402.

[21] C. Park, J. Yoon, E.L. Thomas, Enabling nanotechnology with self assembled block copolymer patterns, Polymer 44(22) (2003) 6725-6760. 
[22] X. Gu, I. Gunkel, A. Hexemer, W. Gu, T.P. Russell, An In Situ Grazing Incidence X-Ray Scattering Study of Block Copolymer Thin Films During Solvent Vapor Annealing, Adv. Mater. (Weinheim, Ger.) 26(2) (2014) 273-281.

[23] C. Sinturel, M. Vayer, M. Morris, M.A. Hillmyer, Solvent Vapor Annealing of Block Polymer Thin Films, Macromolecules (Washington, DC, U. S.) 46(14) (2013) 5399-5415.

[24] L. Wan, S. Ji, C.-C. Liu, G.S.W. Craig, P.F. Nealey, Directed self-assembly of solventvapor-induced non-bulk block copolymer morphologies on nanopatterned substrates, Soft Matter 12(11) (2016) 2914-2922.

[25] J.N.L. Albert, W.-S. Young, R.L. Lewis, T.D. Bogart, J.R. Smith, T.H. Epps, Systematic Study on the Effect of Solvent Removal Rate on the Morphology of Solvent Vapor Annealed ABA Triblock Copolymer Thin Films, ACS Nano 6(1) (2012) 459-466.

[26] A.V. Berezkin, C.M. Papadakis, I.I. Potemkin, Vertical Domain Orientation in CylinderForming Diblock Copolymer Films upon Solvent Vapor Annealing, Macromolecules 49(1) (2016) 415-424.

[27] J.G. Son, K.W. Gotrik, C.A. Ross, High-Aspect-Ratio Perpendicular Orientation of PS-bPDMS Thin Films under Solvent Annealing, ACS Macro Letters 1(11) (2012) 1279-1284.

[28] I. Gunkel, X. Gu, Z. Sun, E. Schaible, A. Hexemer, T.P. Russell, An in situ GISAXS study of selective solvent vapor annealing in thin block copolymer films: Symmetry breaking of in-plane sphere order upon deswelling, J. Polym. Sci. Part B: Polym. Phys. 54(2) (2016) 331-338.

[29] W.I. Park, Y.J. Choi, J.M. Yun, S.W. Hong, Y.S. Jung, K.H. Kim, Enhancing the Directed Self-assembly Kinetics of Block Copolymers Using Binary Solvent Mixtures, ACS Applied Materials \& Interfaces 7(46) (2015) 25843-25850.

[30] J.M. Kim, Y.J. Kim, W.I. Park, Y.H. Hur, J.W. Jeong, D.M. Sim, K.M. Baek, J.H. Lee, M.-J. Kim, Y.S. Jung, Eliminating the Trade-Off between the Throughput and Pattern Quality of Sub-15 nm Directed Self-Assembly via Warm Solvent Annealing, Adv. Funct. Mater. 25(2) (2015) 306-315.

[31] M.A. Chavis, D.-M. Smilgies, U.B. Wiesner, C.K. Ober, Widely Tunable Morphologies in Block Copolymer Thin Films Through Solvent Vapor Annealing Using Mixtures of Selective Solvents, Adv. Funct. Mater. 25(20) (2015) 3057-3065.

[32] W. Bai, K.G. Yager, C.A. Ross, In Situ Characterization of the Self-Assembly of a Polystyrene-Polydimethylsiloxane Block Copolymer during Solvent Vapor Annealing, Macromolecules (Washington, DC, U. S.) 48(23) (2015) 8574-8584.

[33] W.I. Park, S. Tong, Y. Liu, I.W. Jung, A. Roelofs, S. Hong, Tunable and rapid selfassembly of block copolymers using mixed solvent vapors, Nanoscale 6(24) (2014) 1521615221.

[34] Z. Di, D. Posselt, D.-M. Smilgies, R. Li, M. Rauscher, I.I. Potemkin, C.M. Papadakis, Stepwise Swelling of a Thin Film of Lamellae-Forming Poly(styrene-b-butadiene) in Cyclohexane Vapor, Macromolecules 45(12) (2012) 5185-5195.

[35] J.W. Jeong, W.I. Park, M.-J. Kim, C.A. Ross, Y.S. Jung, Highly Tunable Self-Assembled Nanostructures from a Poly(2-vinylpyridine-b-dimethylsiloxane) Block Copolymer, Nano Letters 11(10) (2011) 4095-4101.

[36] K.A. Cavicchi, K.J. Berthiaume, T.P. Russell, Solvent annealing thin films of poly(isoprene-b-lactide), Polymer 46(25) (2005) 11635-11639.

[37] Y. Tada, H. Yoshida, Y. Ishida, T. Hirai, J.K. Bosworth, E. Dobisz, R. Ruiz, M. Takenaka, T. Hayakawa, H. Hasegawa, Directed Self-Assembly of POSS Containing Block Copolymer on Lithographically Defined Chemical Template with Morphology Control by Solvent Vapor, Macromolecules (Washington, DC, U. S.) 45(1) (2012) 292-304. 
[38] I. Tokarev, R. Krenek, Y. Burkov, D. Schmeisser, A. Sidorenko, S. Minko, M. Stamm, Microphase Separation in Thin Films of Poly(styrene-block-4-vinylpyridine) Copolymer-2(4‘-Hydroxybenzeneazo)benzoic Acid Assembly, Macromolecules 38(2) (2005) 507-516.

[39] E.B. Gowd, B. Marcus, S. Manfred, In Situ GISAXS Study on Solvent Vapour Induced Orientation Switching in PS- b -P4VP Block Copolymer Thin Films, IOP Conference Series: Materials Science and Engineering 14(1) (2010) 012015.

[40] J.C. Brendel, F. Liu, A.S. Lang, T.P. Russell, M. Thelakkat, Macroscopic Vertical Alignment of Nanodomains in Thin Films of Semiconductor Amphiphilic Block Copolymers, ACS Nano 7(7) (2013) 6069-6078.

[41] S. Mondal, S.R. Wickramasinghe, Photo-induced graft polymerization of N-isopropyl acrylamide on thin film composite membrane: Produced water treatment and antifouling properties, Separation and Purification Technology 90 (2012) 231-238.

[42] R.P. F. Montagne, A. M. Popa, A. Hoogerwerf, T. Overstolz, H. Heinzelmann, StimuliResponsive Polymers in MEMS Devices, NSTI-Nanotech 2008, pp. 653-656.

[43] I. Tokarev, S. Minko, Stimuli-responsive hydrogel thin films, Soft Matter 5(3) (2009) 511-524.

[44] X. Wang, X. Wang, R. Fernandez, M. Yan, A.L. Rosa, Nanolithography on responsive materials, Journal of Nanoscience Letters 2(23) (2012) 1-16.

[45] L. Rusen, V. Dinca, B. Mitu, C. Mustaciosu, M. Dinescu, Temperature responsive functional polymeric thin films obtained by matrix assisted pulsed laser evaporation for cells attachment-detachment study, Applied Surface Science 302 (2014) 134-140.

[46] C. Xu, X. Fu, M. Fryd, S. Xu, B.B. Wayland, K.I. Winey, R.J. Composto, Reversible Stimuli-Responsive Nanostructures Assembled from Amphiphilic Block Copolymers, Nano Letters 6(2) (2006) 282-287.

[47] S. Wu, C. Bubeck, Macro- and Microphase Separation in Block Copolymer Supramolecular Assemblies Induced by Solvent Annealing, Macromolecules (Washington, DC, U. S.) 46(9) (2013) 3512-3518.

[48] X. Li, J. Peng, Y. Wen, D.H. Kim, W. Knoll, Morphology change of asymmetric diblock copolymer micellar films during solvent annealing, Polymer 48(8) (2007) 2434-2443.

[49] C. Liang, K. Hong, G.A. Guiochon, J.W. Mays, S. Dai, Nanoporous films: Synthesis of a large-scale highly ordered porous carbon film by self-assembly of block copolymers, Angewandte Chemie, International Edition 43(43) (2004) 5785-5789.

[50] S.-M. Jeon, S.H. Lee, S.I. Yoo, B.-H. Sohn, Ordered Complex Nanostructures from Bimodal Self-Assemblies of Diblock Copolymer Micelles with Solvent Annealing, Langmuir 27(19) (2011) 12191-12196.

[51] S. Park, J.-Y. Wang, B. Kim, W. Chen, T.P. Russell, Solvent-Induced Transition from Micelles in Solution to Cylindrical Microdomains in Diblock Copolymer Thin Films, Macromolecules 40(25) (2007) 9059-9063.

[52] A. Bousquet, E. Ibarboure, E. Papon, C. Labrugère, J. Rodríguez-Hernández, Structured multistimuli-responsive functional polymer surfaces obtained by interfacial diffusion of amphiphilic block copolymers, J. Polym. Sci. A Polym. Chem. 48(9) (2010) 1952-1961.

[53] C.S. Thomas, L. Xu, B.D. Olsen, Kinetically Controlled Nanostructure Formation in Self-Assembled Globular Protein-Polymer Diblock Copolymers, Biomacromolecules 13(9) (2012) 2781-2792.

[54] Q. Yu, Y. Zhang, H. Chen, F. Zhou, Z. Wu, H. Huang, J.L. Brash, Protein Adsorption and Cell Adhesion/Detachment Behavior on Dual-Responsive Silicon Surfaces Modified with Poly(N-isopropylacrylamide)-block-polystyrene Copolymer, Langmuir 26(11) (2010) 85828588 . 
[55] J.T. Lai, D. Filla, R. Shea, Functional Polymers from Novel Carboxyl-Terminated Trithiocarbonates as Highly Efficient RAFT Agents, Macromolecules 35(18) (2002) 67546756.

[56] H. Tanaka, H. Hasegawa, T. Hashimoto, Ordered structure in mixtures of a block copolymer and homopolymers. 1. Solubilization of low molecular weight homopolymers, Macromolecules 24(1) (1991) 240-251.

[57] H.G. Schild, Poly(N-isopropylacrylamide): experiment, theory and application, Progress in Polymer Science 17(2) (1992) 163-249.

[58] M.Y. Paik, J.K. Bosworth, D.-M. Smilges, E.L. Schwartz, X. Andre, C.K. Ober, Reversible Morphology Control in Block Copolymer Films via Solvent Vapor Processing: An in Situ GISAXS Study, Macromolecules (Washington, DC, U. S.) 43(9) (2010) 4253-4260.

[59] D.R. Lide, Handbook of Organic Solvents, CRC Press, Boca Raton, FL, USA, 1995.

[60] C.M. Hansen, Hansen Solubility Parameters: A User's Handbook, CRC Press, U.S.A, 2000 .

[61] H. Ahmad, Solubility Parameter of Acrylamide Series Polymers through Its Components and Group Contribution Technique, Journal of Macromolecular Science: Part A - Chemistry 17(4) (1982) 585-600.

[62] J. Zhang, D. Posselt, D.-M. Smilgies, J. Perlich, K. Kyriakos, S. Jaksch, C.M. Papadakis, Lamellar Diblock Copolymer Thin Films during Solvent Vapor Annealing Studied by GISAXS: Different Behavior of Parallel and Perpendicular Lamellae, Macromolecules 47(16) (2014) 5711-5718.

[63] W.A. Phillip, M.A. Hillmyer, E.L. Cussler, Cylinder Orientation Mechanism in Block Copolymer Thin Films Upon Solvent Evaporation, Macromolecules 43(18) (2010) 77637770 . 
Graphical Abstract
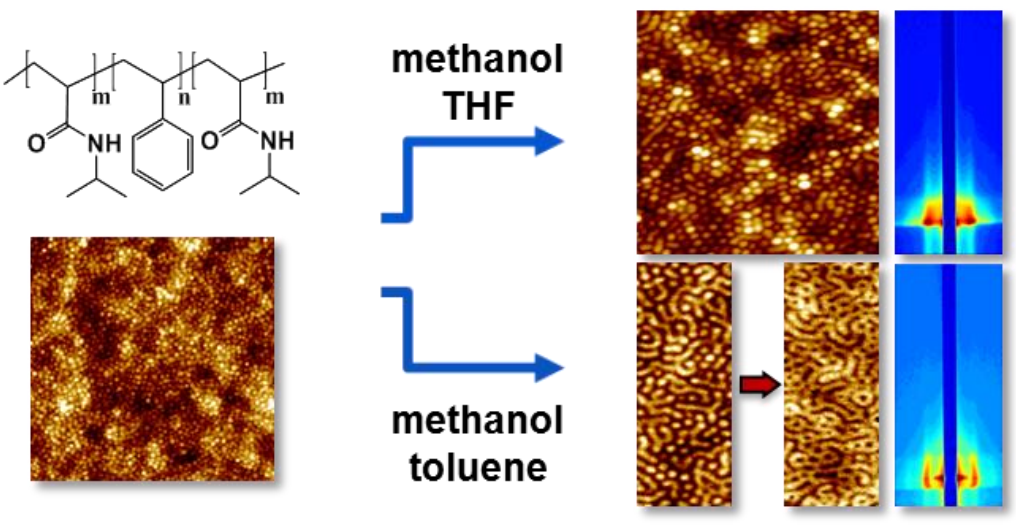